\title{
The diagnostic value of immunohistochemistry in the typing of renal tumors with eosinophylic cytoplasma
}

\author{
Kucuk $\mathrm{S}^{1}$, Akpolat $\mathrm{N}^{2}$ \\ Department of Pathology, Faculty of Medicine, Usak University, Usak, Turkey. ata2012irin53@yahoo.com
}

\begin{abstract}
AIM: In this study, we aimed to review the diagnostic approach to eosinophilic cell renal neoplasms by light microscopy and immunohistochemical techniques.

METHOD: In this study 23 of these tumors were eosinophilic variant classic RCC, 15 eosinophilic variant papillary RCC, 13 eosinophilic variant chromophobe RCC and 13 oncocytoma cases. These tumors were immunohistochemically treated with CK7, CD117, EpCAM, Vimentin, RCCm (Renal cell carcinoma marker) and GST- $\alpha$.

RESULTS: In our study, contrary to the general literature on Vimentin, $65.2 \%$ negativity was found in our patients with eosinophilic variant classic RCC. However, when compared with other tumor types in our study, vimentin expression was highest in eosinophilic variant classical RCC with $34.8 \%$. Statistically; RCCm, GST- $\alpha$, EpCAM, CD117, CK7 were found to be significantly associated with tumor types, while no significant relationship was found between Vimentin and tumor types. RCCm positivity and CK7 and CD117 negativity were in favour of eosinophilic variant classical RCC, EpCAM, CK7 and CD117 positivity and Vimentin, GST-a and RCCm negativity supported eosinophilic variant chromophobe RCC, CK7 and RCCm positivity and CD117 and GST- $\alpha$ negativity were found in favour of eosinophilic variant papillary RCC. CD117 positivity and Vimentin, CK7 and GST- $\alpha$ negativity were found to support oncocytoma.

CONCLUSIONS: The panel with RCCm, GST- $\alpha$, EpCAM, CD117, CK7 will contribute to the differentiation of eosinophilic cytoplasm renal tumors that cannot be determined by morphological findings and to reach the correct diagnosis (Tab. 3, Fig. 4, Ref. 54). Text in PDF www.elis.sk

KEY WORDS: RCC, oncocytoma, immunohistochemistry.
\end{abstract}

\section{Introduction}

Following the prostate and bladder carcinomas, the RCC is the third most frequent urological malignity; it is the most lethal urological cancer with poor prognosis and constitutes $2 \%$ of all the types of cancer (1-7). RCC might remain asymptomatic until advanced/metastatic phase. Metastatic diseases constitute approximately one-third of RCC patients and $50 \%$ of them are detected by coincidence in imaging tests performed because of unrelated reasons (7). After the surgical resection, metastases are observed in one-third of RCC patients and recurrence in $40 \%(1-10)$. The incidence of RCC increases in 6th and 7th decades and the male/ /female ratio is $2-2.5 / 1(10-18)$.

It is generally known that RCC is resistant to chemotherapy $(6,7)$. Besides that, significant advancements were achieved in molecular goal-directed therapies for specific RCC types with well-defined histology and molecular anomalies. For this reason,

\footnotetext{
${ }^{1}$ Department of Pathology, Faculty of Medicine, Usak University, Usak, Turkey, and ${ }^{2}$ Department of Pathology, Faculty of Medicine Inönü University, Malatya, Turkey
}

Address for correspondence: S. Kucuk, MD, Department of Pathology, Faculty of Medicine, Usak University, 1 Eylul Campus, 64000, Usak, Turkey.

Phone: +90.05056831285 the accurate histological diagnosis and classification gained more importance than before (6). Although many renal tumors are morphologically diagnosed, there also are certain overlapping morphological characteristics such as benign masqueraders and newly emerging tumor types. Such diagnostic problems are especially observed in small biopsies, metastatic RCC (7), eosinophilic variants of RCC as in the present study, and in oncocytoma cases. Because of its prognostic importance, these tumors should be distinguished and the immunohistochemical panels must be utilized in the differential diagnosis (1-3). In the present study, the primary antibodies CK7, CD117, EpCAM, vimentin, RCCm, and GST- $\alpha$ were immunohistochemically applied to the eosinophilic variant classic RCC, eosinophilic variant papillary RCC, eosinophilic-granular variant of chromophobe RCC, and oncocytoma cases. The diagnostic value of these markers was investigated for these tumors.

\section{Methods}

\section{Study design and data collection}

Sixty-four nephrectomy materials obtained from the archive of the Pathology Department of Medical Faculty, Firat University, were retrospectively involved in the present study. Of these tumors, 23 were eosinophilic variant classic RCC, 15 eosinophilic variant papillary RCC, 13 eosinophilic variant chro- 
Tab. 1. Results of immunohistochemical analysis.

\begin{tabular}{lccccc}
\hline & Clone & Concentration & $\begin{array}{c}\text { Application } \\
\text { time }\end{array}$ & Retrieval & $\begin{array}{c}\text { Manufacturer and place } \\
\text { or production }\end{array}$ \\
\hline CD 117 & K44.2 & $1 / 20$ & $45 \mathrm{~min}$ & Citrate buffer & lab vision, USA \\
CK7 & OV-TL,12/30 & $1 / 50$ & $45 \mathrm{~min}$ & Citrate buffer & Invitrogen, DAKO, USA \\
EpCAM & AUA1 & $1 / 50$ & $45 \mathrm{~min}$ & Citrate buffer & Thermo, USA \\
GST-a & & $1 / 50$ & $45 \mathrm{~min}$ & Citrate buffer & Novacastra, USA \\
Vimentin & V9 & $1 / 20$ & $45 \mathrm{~min}$ & Citrate buffer & Thermo, USA \\
RCCm & PN-15 & $1 / 20$ & $45 \mathrm{~min}$ & Citrate buffer & Thermo, USA \\
\hline
\end{tabular}

$2=$ Medium $(11-50 \%)$, and $3=$ Diffuse $(>50 \%)$.

\section{Ethical considerations}

The study was performed in accordance with the principles outlined in the Declaration of Helsinki. Ethical approval was received from the local Human Non-invasive Clinical Research Ethics Committee. The mophobe RCC, and 13 oncocytoma cases. Regardless of the previous diagnoses, their slides were reviewed and the final diagnoses were taken as basis in this study. The tumor blocks selected for immunohistochemical staining process were cut into 4-micron thickness and taken to the glasses coated with polylysine. The primary antibodies used were CD117 (lab vision, USA), CK7 (Invitrogen,DAKO), USA, EpCAM (Thermo, USA), GST- $\alpha$ (Novacastra, USA), vimentin (Thermo, USA), and RCCm (Thermo, USA). In immunohistochemical staining process, the sections were kept in a drying oven at $37^{\circ} \mathrm{C}$ for 15 minutes and then passed through xylol and alcohol series for 5 minutes each. Then, they were rinsed with distilled water. The sections were kept in $3 \%$ hydrogen peroxide for 10 minutes in order to prevent endogen peroxidase activity and then rinsed again with distilled water for 1 minute. Then, for antigen retrieval process, the sections were boiled with $10 \%$ citrate buffer ( $\mathrm{pH}$ 6.0) for three times ( 5 minutes each) in a microwave oven at $750 \mathrm{MW}$. Then, they were left for cooling at room temperature for 20 minutes. Following this step, they were rinsed in PBS for 5 minutes. Then, the sections were dried and the tissues were circumscribed using pap-pen. For the protein blockage, Blocking Reagent-Ultra V Blok (Thermo, USA) was dripped and kept in bain-marie for 10 minutes. The fluid in the sections was removed by shaking the glasses. The residuals were removed by drying. The primary antibodies were then applied and the details about the primary antibodies are presented in Table 1 . The primary antibodies CD117, CK7, EpCAM, GST- $\alpha$, and Vimentin were incubated with RCC for 45 minutes.

They were rinsed with PBS for 5 minutes. The residuals were dried and the secondary antibody Value HRP Polymer was incubated in bain-marie for 20 minutes. After rinsing in PBS for 5 minutes, the residuals were dried. Then, they were incubated with chromogen (AEC chromogen) for 10 minutes and rinsed with tap water. After contrast staining with Mayers hematoxylin for 2 minutes, the specimens were dehydrated and coated with Ultramount.

As a control, the bladder tumor in CK7, GIST in CD117, ligament tissues in Vimentin, normal renal tissues in RCCm and GST- $\alpha$, and breast tumor in EpCAM were used. The preparations were examined using Olympus BX51 light microscope. The cytoplasmic staining pattern was taken into consideration in CK7 and Vimentin, membranous-cytoplasmic pattern in RCC and CD117, membranous pattern in EpCAM, and nuclear and/or cytoplasmic pattern in GST- $\alpha$. The staining density of positively stained tumor cells was divided into 4 sub-groups. Accordingly, the scoring was performed as follows: $0=$ Negative $(<5 \%), 1=$ Focal $(5-10 \%)$, informed consent was not requested since the study was retrospective, the data were analyzed anonymously.

\section{Statistical analysis}

The statistical analyses were performed using SPSS-12.0 software by calculating the Chi-Square and Correlation tests. The statistical significance was set at $\mathrm{p}<0.05$.

\section{Results}

When using RCCm stain in 23 eosinophilic variant classic RCC cases, 14 (60.9\%) diffuse, 4 (17.4\%) medium, 2 (8.7\%) focal and 3 (13\%) negative staining were observed (Fig. 1a). Among fifteen eosinophilic variant papillary RCC cases, there were 12 (80\%) diffuse and 2 (13.3\%) medium staining (Fig. 3a). Of thirteen eosinophilic variant chromophobe RCC cases, only 1 (7.7 \%) was diffusely stained and there was no medium or focal staining (Fig. 2a). Moreover, none of the 13 oncocytoma cases was observed as positive (Fig. 4a). A statistically significant relationship was found between RCCm and tumor types $(p=0)$.

\section{CK7}

When using CK7 stain in 23 eosinophilic variant classic RCC cases, there was $1(4.3 \%)$ medium staining, but no staining was observed in 22 cases (95.7 \%) (Fig. 1b). Of fifteen eosinophilic variant papillary RCC cases, 9 (60\%) were diffusely stained, 2 $(13.3 \%)$ were stained at medium level, $1(6.7 \%)$ was stained focally, but no staining was observed in 3 (20\%) of cases (Fig. $3 \mathrm{~b})$. Of thirteen eosinophilic variant chromophobe RCC cases, 10 $(76.9 \%)$ cases were stained diffusely and $2(15.4 \%)$ at medium level, but no staining was observed in 1 (7.7 \%) case (Fig. 2b). Of thirteen oncocytoma cases, there was $1(7.7 \%)$ with medium level staining, but no staining was observed in twelve (92.3\%) cases (Fig. 4b). A statistically significant relationship was found between CK7 and tumor types $(p=0)$.

\section{CD117}

When using CD117 stain in 23 eosinophilic variant classic RCC cases, it was observed that one (4.3\%) case was diffusely stained and $2(8.7 \%)$ cases were focally stained, but no staining was observed in 20 (87\%) cases (Fig. 1b). Among fifteen eosinophilic variant papillary RCC cases, there were 1 (6.7\%) diffuse, 1 (6.7\%) medium, and 1 (6.7\%) focal staining but no staining was observed in 12 (80\%) cases (Fig. 3b). Among thirteen eosinophilic

\section{Immunohistochemical staining characteristics $\mathrm{RCCm}$}




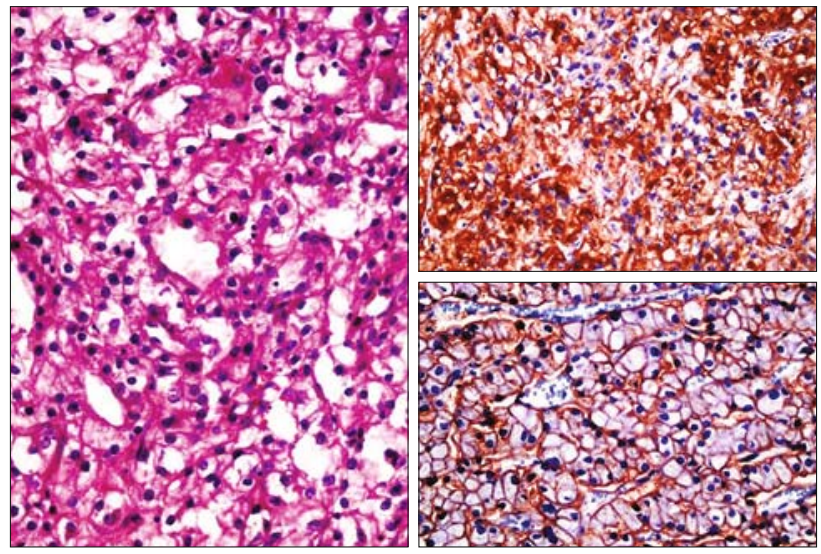

Fig. 1a. Eosinophilic variant classic RCC; left eosinophilic variant of the classic RCC histopathological image (H \& Ex400) on the left, RCCm positivity in the upper right corner and EPCAM positivity in the lower right corner (immunoperoxidase, $x 400$ ).
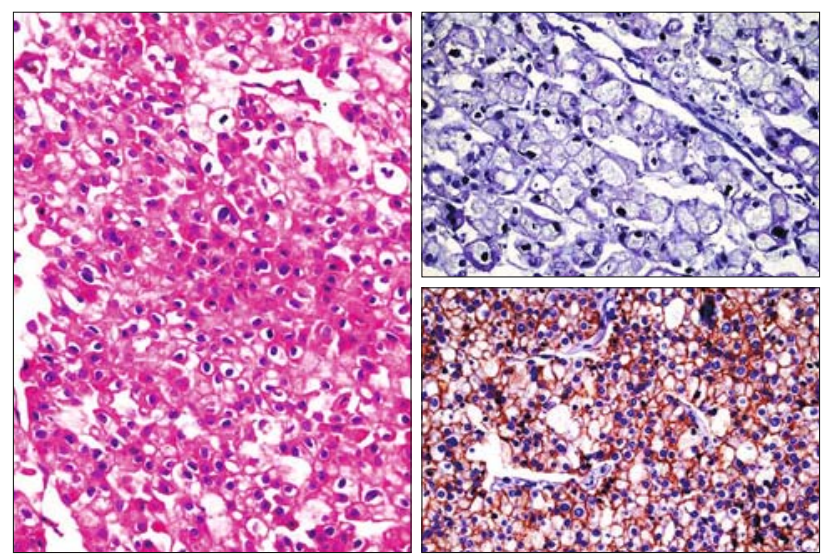

Fig. 2a. Eosinophilic variant chromophobe RCC; histopathological image of eosinophilic variant chromophobe RCC (H \& Ex400) on the left, negativity of RCCm in the upper right corner, and EpCAM positivity in the lower right corner (immunoperoxidase, $x 400$ ).
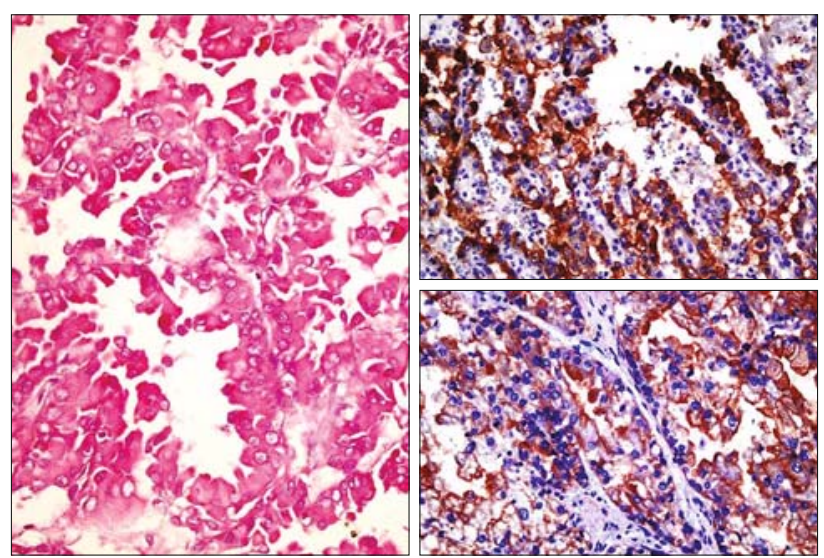

Fig. 3a. Eosinophilic variant papillary RCC; histopathological appearance of esinophilic variant papillary RCC (H \& Ex400) on the left, positivity of RCCm in the upper right corner, and EpCAM positivity in the lower right corner (immunoperoxidase, $\mathbf{x} 400$ ).
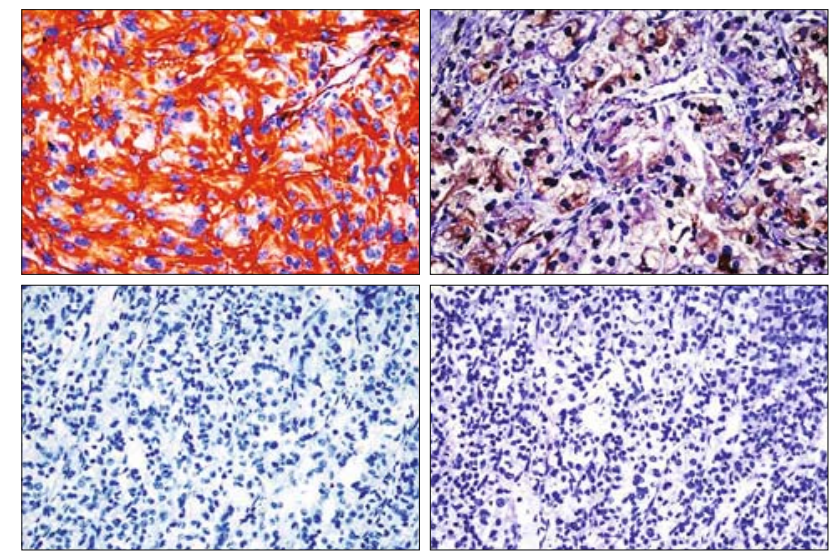

Fig. 1b. Eosinophilic variant classic RCC; vimentin positivity of classic RCC in upper left corner, GST- $\alpha$ positivity in upper right corner, CD117 negativity in lower left corner, CK7 negativity in lower right corner (immunoperoxidase, $\mathbf{x 4 0 0 )}$ ).

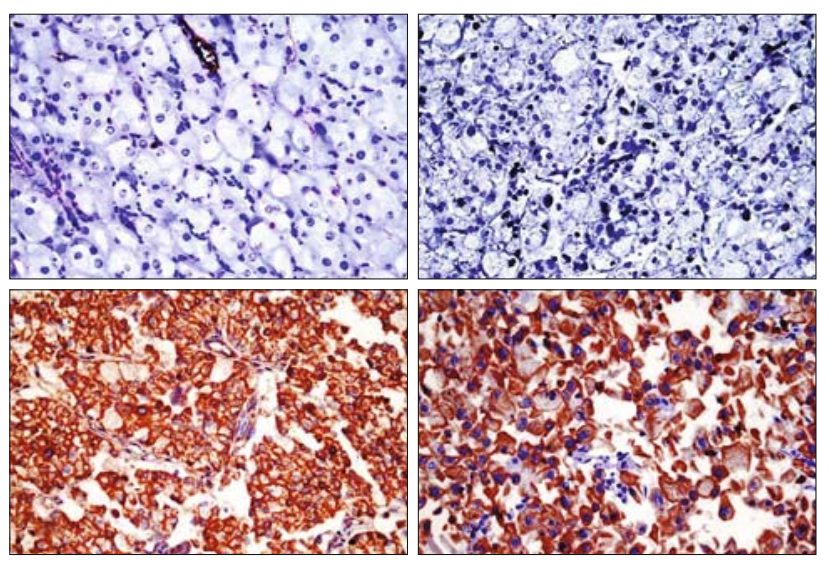

Fig. 2b. Eosinophilic variant chromophobe RCC; vimentin negativity in the upper left corner, GST- $\alpha$ negativity in the upper right corner, CD117 positivity in the lower left corner, CK7 positivity in the lower right corner (immunoperoxidase, $x 400$ ).
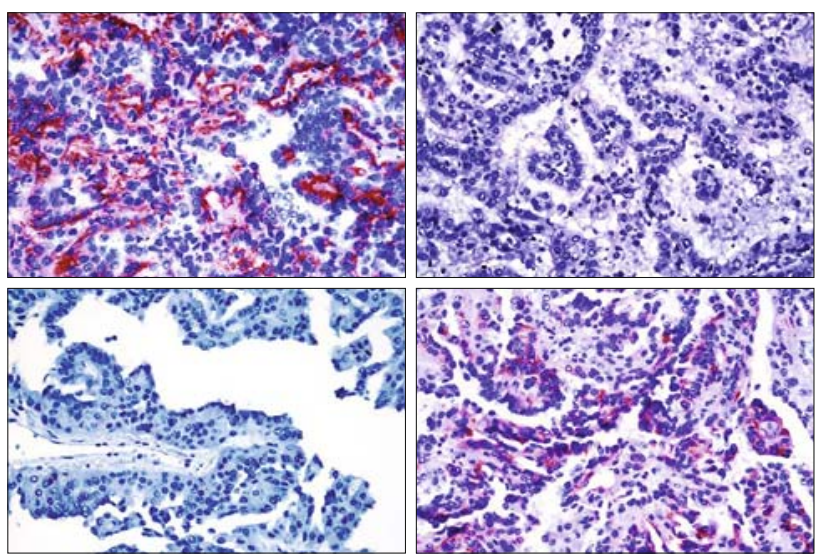

Fig. 3b. Eosinophilic variant papillary RCC; Vimentin negativity in the upper left corner eosinophilic variant papillary RCC, GST- $\alpha$ negativity in the upper right corner, CD117 negativity in the lower left corner and positivity of $\mathrm{CK} 7$ in the lower right corner (immunoperoxidase, $\mathbf{x 4 0 0}$ ). 


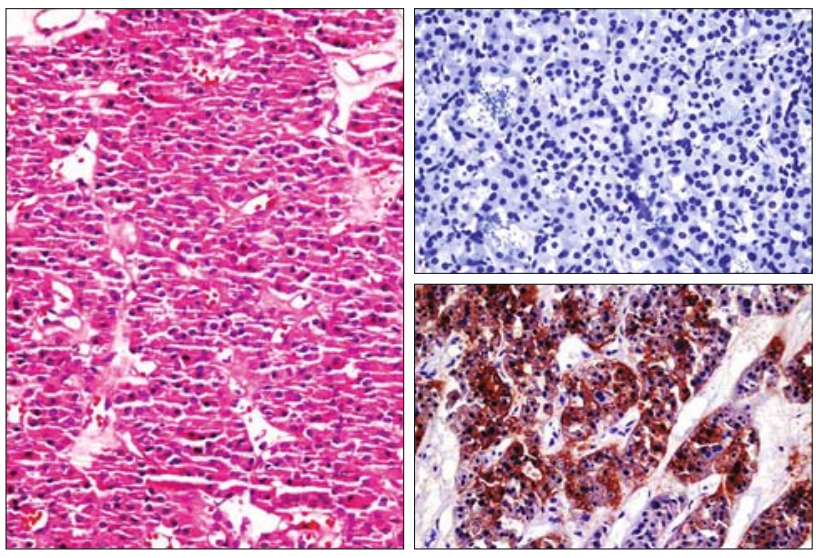

Fig. 4a. Oncocytoma; Oncocytoma shows histopathological appearance (H\& Ex400) on the left, RCCm negativity in the upper right corner and EpCAM positivity in the lower right corner (immunoperoxidase, $\mathbf{x} 400$ ).

variant chromophobe RCC cases, there were 6 (46.2 \%) diffuse, $4(30.8 \%)$ medium, and 1 (7.7 \%) focal staining, but no staining was observed in $2(15.4 \%)$ cases (Fig. 2b). Of thirteen oncocytoma cases, 8 (61.5\%) were stained diffusely and 4 (30.8\%) were stained at medium level, but no staining was observed in 1 (7.7\%) case (Fig. 4b). A statistically significant relationship was found between CD117 and tumor types $(\mathrm{p}=0)$.

\section{EpCAM}

When using EpCAM stain in 23 eosinophilic variant classic RCC cases, it was determined that $2(8.7 \%)$ of cases were diffusely stained, $5(21.7 \%)$ at medium level and $6(26.1 \%)$ at focal level, whereas no staining was observed in $10(43.5 \%)$ cases (Fig. 1a). Of fifteen eosinophilic variant papillary RCC cases, 6 (40\%) were diffusely stained, 2 (13.3\%) at medium level and $2(13.3 \%)$ at focal level but no staining was observed in 5 (33.3\%) cases (Fig. $3 a)$. The staining was observed in all 13 eosinophilic variant chromophobe RCC cases (Fig. 2a). Among thirteen oncocytoma cases, there were $3(23.1 \%)$ medium level and $2(15.4 \%)$ focal staining, but no staining was observed in $8(61.5 \%)$ cases (Fig. 4a). A statistically significant relationship was found between EpCAM and tumor types $(\mathrm{p}=0.009)$

\section{GST- $\alpha$}

When using GST- $\alpha$ stain in 23 eosinophilic variant classic RCC cases, it was observed that there were 7 (30.4\%) diffuse, 4 (17.4\%) medium level, and $2(8.7 \%)$ focal staining, but no staining was observed in 10 (43.5\%) cases (Fig. 1b). Among fifteen eosinophilic variant papillary RCC cases (Fig. 3b) and thirteen eosinophilic variant chromophobe RCC cases (Fig. 2b), there was no staining. Among thirteen oncocytoma cases, there was 1 (7.7 $\%$ ) diffuse staining but no staining was observed in 12 (92.3\%) cases (Fig. 4b). A statistically significant relationship was found between GST- $\alpha$ and tumor types $(\mathrm{p}=0)$.

\section{Vimentin}

When using Vimentin in 23 eosinophilic variant classic RCC

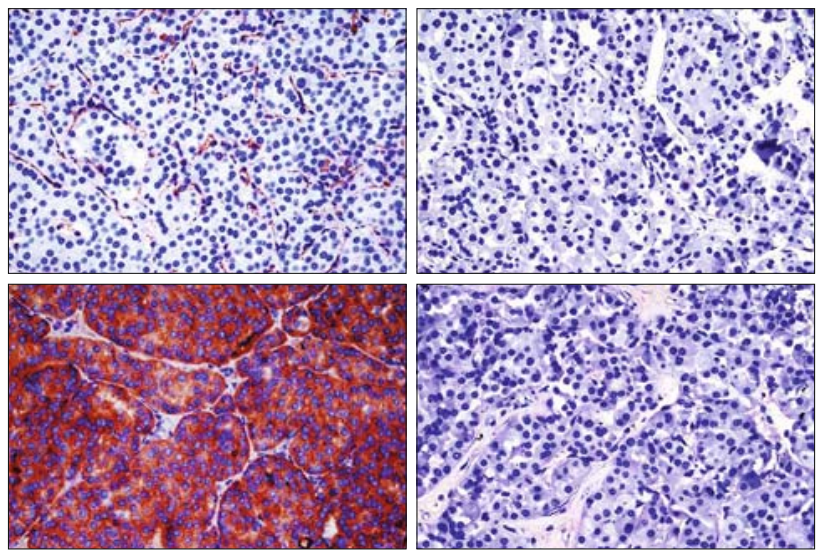

Fig. 4b. Oncocytoma; vimentin negativity in the left upper corner, GST- $\alpha$ negativity in the upper right corner, positivity of CD117 in the lower left corner and CK7 negativity in the lower right corner (immunoperoxidase, $x 400$ ).

cases, it was determined that there were $3(13 \%)$ diffuse staining, 2 (8.7\%) medium level staining, and 3 (13\%) focal staining, but no staining was observed in 15 (65.2\%) cases (Fig. 1b). Among fifteen eosinophilic variant papillary RCC cases, there were 2 (13.3\%) diffuse, 2 (13.3\%) medium level, and 2 (13.3 \%) focal staining, but no staining was observed in 9 (60\%) cases (Fig. 3b). One ( $7.7 \%)$ of thirteen eosinophilic variant chromophobe RCC cases was focally stained, but no staining was observed in 12 (92.3\%) cases (Fig. 2b). No staining was observed in thirteen oncocytoma cases (Fig. 4b). No statistically significant relationship was found between Vimentin and tumor types $(\mathrm{p}=0.21)$.

The staining percentages of tumor subtypes in CK7, RCCm, GST- $\alpha$, Vimentin, CD117, and EpCAM are presented in Table 2 , whereas the diagnostic value of negativity and positivity of markers used in the differential diagnosis of tumor subtypes is presented in Table 3.

\section{Discussion}

Given the difficulties in differential diagnosis of eosinophilic cytoplasmic epithelial renal tumors, the difficulty of diagnosis becomes more prominent in cases with limited cellular specimens such as "tru-cut" biopsy and fine-needle aspiration cytology, as well as intraoperative (frozen section) examinations (7, 19-31). In such cases, the use of "oncocytic tumor" term is recommended (19-31).

Rather than the expensive and specific methods such as molecular, genetic, and immunohistochemical (IHC) methods in diagnosing the eosinophilic/granular cytoplasmic epithelial tumors of kidney, the macroscopic and microscopic examination is considered to be the golden standard. The macroscopic characteristics of tumor in nephrectomy specimens are useful in diagnosis, as well as it allows multiple specimens from different locations. However, the fine-needle and tru-cut biopsy methods and cytological specimens do not offer this option. These methods necessitate making a diagnosis with minimum one tissue/cell in the hand (7, 29-32). Given the fact that oncocytoma and eosinophilic variant chromophobe RCC might have similar nuclear, cytoplasmic, and 
Tab. 2. Staining of tumor subtypes in CK7, RCCm, GST- $\alpha$, Vimentin, CD117, and EpCAM.

\begin{tabular}{|c|c|c|c|c|c|c|c|c|c|c|c|c|}
\hline \multirow[t]{2}{*}{ Tumor type } & \multicolumn{2}{|c|}{ CK7 } & \multicolumn{2}{|c|}{ RCCm } & \multicolumn{2}{|c|}{ GST- $\alpha$} & \multicolumn{2}{|c|}{ Vimentin } & \multicolumn{2}{|c|}{ CD117 } & \multicolumn{2}{|c|}{ EpCAM } \\
\hline & + & - & + & - & + & - & + & - & + & - & + & - \\
\hline Eosinophilic variant Classic RCC (\%) & 4.3 & 95.7 & 87 & 13 & 56.5 & 43.5 & 34.8 & 65.2 & 13 & 87 & 56.5 & 43.5 \\
\hline Eosinophilic variant Chromophobe RCC (\%) & 92.3 & 7.7 & 7.7 & 92.3 & 0 & 100 & 7.7 & 92.3 & 84.6 & 15.4 & 100 & 0 \\
\hline Eosinophilic variant Papillary RCC (\%) & 80 & 20 & 93.3 & 6.7 & 0 & 100 & 40 & 60 & 20 & 80 & 66.7 & 33.3 \\
\hline Oncocytoma $(\%)$ & 7.7 & 92.3 & 23.1 & 76.9 & 7.7 & 92.3 & 0 & 100 & 92.3 & 7.7 & 38.5 & 61.5 \\
\hline
\end{tabular}

RCC - Renal cell carcinoma

Tab. 3. Diagnostic value of negativity and positivity of markers used in the differential diagnosis of tumor subtypes.

\begin{tabular}{lcccc}
\hline & $\begin{array}{c}\text { Eosinophilic variant } \\
\text { Classic RCC }\end{array}$ & $\begin{array}{c}\text { Eosinophilic variant } \\
\text { Chromophobe RCC }\end{array}$ & $\begin{array}{c}\text { Eosinophilic } \\
\text { variant Papillary RCC }\end{array}$ & Oncocytoma \\
\hline Positive & RCCm & CD117 & CK7 & CD117 \\
& & CK7 & RCCm & \\
\hline Negative & EKCAM & Vimentin & CD117 & CK7 \\
& CD117 & RCCm & GST- $\alpha$ & GST- $\alpha$ \\
& & GST- $\alpha$ & & Vimentin \\
\hline
\end{tabular}

few loops of Henle. With these properties, GST- $\alpha$ is used in the differential diagnosis of renal tumors (18). In studies carried out on this subject, high levels of positivity were reported in classic RCC cases, whereas no or low level staining was reported in chromophobe RCC, papillary RCC and oncocytoma cases $(18,30,39)$. In the present study, no staining was observed in eosinophilic variant papillary and chromophobe

stromal characteristics, the importance of IHC in distinguishing the eosinophilic cell epithelial renal tumors, in which the discrimination cannot be made using morphological diagnostic criteria is higher (7, 29-32).

CD117 is secreted in normal adult renal parenchyma. The negativity of this antibody in classic RCC cases is useful in distinguishing from the other RCCs. However, because of the positivity of this marker in both oncocytoma and eosinophilic variant chromophobe RCC it is useless in distinguishing these two tumors $(10,30,33)$. In studies carried out using CD117, up to $100 \%$ positivity was found in chromophobe RCC and oncocytoma cases, whereas no staining was observed in classic and papillary RCC cases $(17,30$, 33-35). In the present study, although there are certain differences with literature in terms of eosinophilic variant classic and papillary RCC cases, the staining percentages of other tumor subtypes overlap in the literature.

The monoclonal cytokeratins such as CK7 and CK20 stain different components of renal tubular system and are useful in defining the origins of different tumors that might be observed in kidneys. It was claimed that CK7 might be used in the differential diagnosis of oncocytoma and eosinophilic variant of RCC (30, 36,3 7). Although it is generally negative in classic RCC, CK7 was reported to be positive in $11 \%$ in some studies. Extensive positivity was reported in papillary and chromophobe RCCs (30, $33,34,38)$. The positivity in oncocytoma is focal/cytoplasmic and not strongly membranous staining as in chromophobe RCC. Kiremitçi et al reported that the CK7 immune staining density decreases as the nuclear level increases in chromophobe RCC, papillary RCC, and classic RCC cases (12). The results obtained in the present study corroborate with the literature. In the present study, a high level of negativity was detected in eosinophilic variant classic RCC and oncocytoma cases $(95.7 \%$ and $92.3 \%$, respectively), but an immune-reactivity was detected in $84.6 \%$ of eosinophilic variant chromophobe RCC cases.

Significant immune-reactivity was reported with glutathione S-transferase (GST) in the proximal tubule, classic RCC, and
RCC cases, and negativity was found to be $92.3 \%$ in oncocytoma cases, whereas positivity detected in eosinophilic variant classic RCC cases was $56.5 \%$.

EpCAM is immune-reactive in high levels of chromophobe RCCs, whereas it is positive in lower levels of classic RCCS and single/small group oncocytoma cases. It is very useful especially in distinguishing chromophobe RCC and oncocytoma (30, 40-42). However, EpCAM is negative in sarcomatoid chromophobe RCC (30). In the present study, although there are similarities between EpCAM positivity in eosinophilic variant chromophobe RCC cases and literature, there also are significant differences between the positivity percentages in tumor subtypes.

Vimentin is positive especially in classic and papillary RCC cases. It is not expected to be positive in chromophobe RCC and oncocytoma cases (43). Liu et al claimed that vimentin is the most sensitive and most specific marker for classic RCC (30). In various studies, the vimentin positivity was reported to be $51-85 \%$ in classic RCC, $21 \%$ in chromophobe RCC, 80-86 \% in papillary RCC, and $0-20 \%$ in oncocytoma $(34,38,43-46)$. In the present study, the positivity of vimentin was found to be $35 \%$ in eosinophilic variant classic RCC, $40 \%$ in eosinophilic variant papillary RCC, and $8 \%$ in eosinophilic variant chromophobe RCC, whereas no immune reactivity was observed in oncocytoma cases. No statistically significant relationship was found between tumor types and vimentin $(p>0.05)$ and, on the contrary with many studies in literature, a high rate of negativity ( $65 \%$ ) was determined in eosinophilic variant classic RCC. Similar to the present study, Williams et al reported the negativity of vimentin in classic RCC to be $61 \%$ (47).

$\mathrm{RCCm}$ is secreted from the brush borders of renal proximal tubules and has high specificity primarily for classic RCC (48-51). Wang et al reported staining with RCC marker in $80 \%$ of the granular-eosinophilic variant of classic RCC and immune-reactivity in oncocytoma and chromophobe RCC cases (17). Allory et al however, reported positivity at $37 \%$ in classic RCC, $53 \%$ in papillary $\mathrm{RCC}$, and $2 \%$ in oncocytoma, whereas they reported no positivity in chromophobe RCC (34). In the present study, the immune-re- 
activity was detected in $87 \%$ in the eosinophilic variant of classic RCC, $93.3 \%$ in eosinophilic variant papillary RCC, $7.7 \%$ in eosinophilic variant chromophobe RCC, and $23.1 \%$ in oncocytoma.

The difference between the studies is related to the differences in laboratory staining procedures and the use of different antibodies (30). The diagnosis of typical oncocytoma is made generally using $\mathrm{H} \& \mathrm{E}$ staining and no differential diagnosis problem is observed $(22-27,52)$. However, there may differential diagnosis problems between atypical oncocytoma, chromophobe RCC, classic RCC's eosinophilic variant, and eosinophilic variant of papillary RCC. The IHC method is very useful in distinguishing these cases (22-26).

Şen et al emphasized the importance of RCCm and vimentin positivity in eosinophilic variant of classic RCC and the importance of vimentin and RCCm negativity in oncocytoma. It was also reported that $\mathrm{CK} 7$ positivity and vimentin and $\mathrm{RCCm}$ negativity are useful in diagnosis of chromophobe RCC. Even if CD117 can be added to the panel, it was determined in the experiments on radical materials that it is not as reliable as CK7 is in differential diagnosis of oncocytoma and chromophobe RCC. In eosinophilic variant of classic RCC, CK 7 was reported to be negative. In conclusion, Şen et al recommended the use of a panel containing vimentin, CK7, and CD117 in the accurate diagnosis of needle biopsy of kidney (31).

Similarly, Liu et al claimed that the diagnosis would be in favor of chromophobe RCC or oncocytoma in vimentin and GST- $\alpha$ negativity and CD117 positivity and in favor of classic RCC in vimentin positivity and GST- $\alpha$ and CD117 negativity. It was reported that in differential diagnosis of oncocytoma and chromophobe RCC, the CK7 positivity and EpCAM positivity support chromophobe RCC and $\mathrm{CK} 7$ and EpCAM negativity supports the oncocytoma (30).

In many studies, it has been reported that the primary antibodies CD117, EpCAM, GST- $\alpha$, CK7, vimentin, and RCCm are useful in tumors such as chromophobe RCC, oncocytoma, eosinophilic variant of classic RCC, and eosinophilic variant papillary RCC, which morphologically overlapping with each other and causing diagnostic problems. However, the use of a single marker is not useful from diagnostic aspect and it is better to use them as a panel and to evaluate together with the morphology $(30,53)$.

In conclusion, the results obtained in the present study are as follows (Tab. 3):

1.The combination of RCCm positivity and CK7 and CD117 negativity is in favor of eosinophilic variant classic RCC.

2.The combination of EpCAM, CK7, and CD117 positivity and vimentin, GST- $\alpha$, and RCCm negativity is in favor of eosinophilic variant chromophobe RCC.

3.The combination of $\mathrm{CK} 7$ and RCCm positivity and CD117 and GST- $\alpha$ negativity is in favor of eosinophilic variant papillary RCC.

4.The combination of CD117 positivity and vimentin, CK7, and GST- $\alpha$ negativity supports the oncocytoma.

5.The panel as the primary step in RCC with diagnosis problem should include RCCb, CK7, and CD117. In cases, in which the diagnosis cannot be made using this trio, adding vimentin, EpCAM, and GST- $\alpha$ would be very useful.

6.Nowadays, in cases in that the diagnosis cannot be made using morphological results in eosinophilic cytoplasmic cell renal carcinomas with differential diagnosis problem, immunohistochemistry is a very useful diagnostic instrument.

\section{References}

1. Reuter and Tickoo SK. Adult renal tumors. In: Oberman HA, Reuter V, Stoler MH (ed.) Sternberg's diagnostic surgical pathology. Philadelphia Lippıncott Wılliams\& Wılkıns, 2004; 1955-2001.

2. Eble JN, Sauter G, Epstein JI, Sesterhenn IA. Tumors of kidney. Tumors of the urinary system and male genital organs. In World Health Organization Classification of tumors. IARC press: 2004; 9-44.

3. Bonsib SM. Neoplastik Disease of The Kidney. Bostwick DG, Eble JN (ed.) Urologic Surgical Pathology. 2.Bask1, Canada: Mosby 2001; 4-81.

4. Dirim A, Özkardeş H. Böbrek Kanserlerinde İnsidans etyoloji ve Sınıflandırma. Türkiye Klinikleri Üroloji Özel Dergisi 2011; 4: 10-17.

5. Kargı A. Kidney Cancers. Türkiye Klinikleri J Med Onkol-Special Topics 2011; 4 (3): 1-9.

6. Shen SS, Truong LD, Marina Scarpelli M, Antonio Lopez-Beltran. Arch Pathol Lab Med 2012; 136 (4): 410-417.

7. Ozcan A. Immunoprofi les of adult renal epithelial tumors: Immunohistochemistry is still essential for diagnosis of renal tumors (a comprehensive update). J Interdiscipl Histopathol 2015; 3 (3): 81-101.

8. Okon K. Pathology of renal tumors in adults. Molecular biology, histopathological diagnosis and prognosis. Pol J Pathol 2008; 59 (3): 129-176.

9. Rekha PR, Rajendiran S, Rao S, Shroff S, Joseph LD, Prathiba D. Histological reclassification, histochemical characterization and c-kit immunoexpression in renal cell carcinoma. Indian J Urol 2008; 24 (3): 343-347.

10. Krüger S, Sotlar K, Kausch I, Horny HP. Expression of KIT (CD117) in renal cell carcinoma and renal oncocytoma. Oncology 2005; 68 (2-3): 269-275.

11. Young AN, Master VA, Paner GP, Wang MD, Amin BM. Renal Epitelyal Neoplasms: Diagnostic applications of gene expression profiling. Adv. Anat Pathol 2008; 15 (1): 28-38.

12. Kiremitçi $S$, Tulunay $O$, Baltacı $S$, Gögüş $O$. Diagnostic utility of cytokeratins 7, 10 and 20 in renal cell carcinoma and oncocytoma. Tur J Path 2008; 24 (3): 140-146.

13. Kitamura H, Tsukamoto T. Prognostic biomarkers of renal cell carcinoma: Recent advances. Indian J Urol 2008; 24 (1): 10-15.

14. Geramizadeh B, Ravanshad M, Rahsaz M. Useful markers for differential diagnosis of oncocytoma, chromophobe renal cell carcinoma and conventional renal cell carcinoma. Indian J Pathol Microbiol 2008; 51 (2): 167-171.

15. Zigeuner R, Ratschek M, Langner C. Kit (CD117) immunoreactivity is rare in renal cell and upper urinary tract transitional cell carcinomas. BJU International 2004; 95 (3): 315-318.

16. Buzio L, Palma GD, Mozzoni P, Tondel M, Buzio C, Franchini I, Axelson O, Mutti A. Glutathione S-transferases M1-1 and T1-1 as risk modifiers for renal cell cancer associated with occupational exposure to chemicals. Occup Environ Med 2003; 60 (10): 789-793.

17. Wang, You H, Mills, Stacey E. KIT and RCC Are Useful in distinguishing chromophobe renal cell carcinoma from the granular variant of clear cell renal cell carcinoma. Am J Surg Pathol 2005; 29 (5): 640-646.

18. Chuang ST, Chu P, Sugimura J, Tretiakova MS, Papavero V, Wang $K$ et al. Overexpression of Glutathione S-Transferase $\alpha$ in clear cell renal cell carcinoma. Am J Clin Pathol 2005; 123 (3): 421-429.

19. Şen S, Sarkık B, Şimşir A, Kısmalı E, Gökmen E. Needle core biopsies for renal masses and diagnostic difficulties. Türk Patoloji Dergisi 2009; 25 (1): $5-18$ 
20. Shah RB, Rahkshi N, Hafez KS, Wood DP Jr, Kunju LP. Image-guided biopsy in the evaluation of renal mass lesions in contemporary urological practice: indications, adequacy, clinical impact, and limitations of the pathological diagnosis. Hum Pathol 2005; 36 (12): 1309-1315.

21. Harik LR, Pashaei S, Humphrey PA, Tamboli P, Amin MB. Needle core biopsy of the kidney for mass lesions: a clinico-pathologic perspective based on 196 cases. Mod Pathol 2005; 18: 144A.

22. Lebret T, Poulain JE, Molinie V, Herve JM, Denoux Y, Guth A et al. Percutaneous core biopsy for renal masses: indications, accuracy and results. J Urol 2007; 178 (4 Pt 1): 1184-1188.

23. Sofukerim M, Tatlışen A, Canöz Ö, Tokat F. Renal kitlelerinin tanısında perkütan iğne kor biyopsinin rolü nedir? Türk Üroloji Dergisi 2007; 33 (4): 405-410.

24. Skinnider BF, Amin MB. An immunohistochemical approach to the differential diagnosis of renal tumors. Semin Diagn Pathol 2005; 22 (1): 51-68.

25. Skinnider BF, Folpe AL, Hennigar RA, Lim SD, Cohen C, Tamboli $P$ et al. Distribution of cytokeratins and vimentin in adult renal neoplasms and normal renal tissue: potential utility of a cytokeratin antibody panel in the differential diagnosis of renal tumors. Am J Surg Pathol 2005; 29 (6): 747-754.

26. Avery AK, Beckstead J, Renshaw AA, Corless CL. Use of antibodies to RCC and CD10 in the differential diagnosis of renal neoplasms. Am J Surg Pathol 2000; 24 (2): 203-210.

27. Shuch B, Amin A, J Armstrong AJ. Ficarra V, Lopez- Beltran et al. Understanding Pathologic Variants of Renal Cell Carcinoma: Distilling Therapeutic Opportunities from Biologic Complexity. Eur Assoc Urol 2014; 67 (1): 85-97.

28. Kılışaslan I. Pathology of Kidney Tumors. Türkiye Klinikleri J UrologySpecial Topics 2011; 4 (1): 18-25.

29. Mete Ö, Kılıșaslan I, Uysal V. The utılıty of hıstomorphologıcal features in the differentiation of eosinophilic chromophobe renal cell carcinoma and oncocytoma. Tur J Path 2005; 21 (3-4): 62-66.

30. Liu L, Qian J, Singh H, Meiers I, Zhou X, Bostwick DG. Immunohistochemical analysis of chromophobe renal cell carcinoma, renal oncocytoma, and clear cell carcinoma. An optimal and practical panel for differential diagnosis. Arch Pathol Lab Med 2007;131 (8): 1290-1296.

31. Şen S, Sarkık B, Şimşir A. Immunohistochemical Markers in Renal Tumors and Findings in Non-Tumoral Renal Parenchyma 2010; 26 (2): 120-129.

32. Zhang W, Yu WJ, Chen YQ, Kang EH, Liu Y, Zhuang J et al. Diagnostic utility of immunohistochemistry in differential diagnosis of renal tumors with oncocytic features. Zhonghua Bing Li Xue Za Zhi 2016; 45 (10): 692-697.

33. Pan CC, Chen PCH, Chiang H. Overexpression of KIT (CD117) in Chromophobe Renal cell carcinoma and renal oncocytoma. Am J Clin Pathol 2004; 121 (6): 878-883.

34. Allory Y, Bazille C, Vieillefond A, Molinie V, Cochand-Priollet B, Cussenot $\mathrm{O}$ et al. Profiling and classification tree applied to renal epithelial. Histopathology 2008; 52 (2): 158-166.

35. Safinaz H, Shorbagy E, Alshenawy HA. Diagnostic utility of vimentin, CD117, cytokeratin 7 and

36. caveolin-1 in differentiation between clear cell renal cell carcinoma, chromophobe renal cell carcinoma and oncocytoma. J Microscop Ultrastruct 2017; 5 (2): 90-96.

37. Mathers ME, Pollock AM, Marsh C, O'Donnell M. Cytokeratin7: a useful adjunct inthe diagnosis of chromophobe renal cell carcinoma. Histopathol 2002; 40 (6): 563-567.

38. Alexa A, Baderca F, Lighezan R, Izvernariu D, Raica M. The diagnostic value of cytokeratins expression in the renal parenchyma tumors. Roman J Morphol Embryol 2010; 51 (1): 27-35.
39. Pradhan D, Kakkar N, Bal A, Kumar Singh S, Joshi K. Sub-typing of renal cell tumours; contribution of ancillary techniques. Diagn Pathol 2009; 4 (21): 1-8.

40. Takahashi M, Yang XJ, Sugimura J, Backdahl J, Tretiakova M, Qian CN. Molecular subclassification of kidney tumors and the discovery of new diagnostic markers. Oncogene 2003; 22: 6810-6818.

41. Went P, Dirnhofer S, Salvisberg T, Amin M, Lim S, Diener PA, Moch H. Expression of epithelial cell adhesion molecule (EpCam) in renal epithelial tumors. Am J Surg Pathol 2005; 29 (1): 83-88.

42. Rioja J Rioja et al. Epithelial cell a dhesion molecule (EpCAM) expression in renal cell carcinomas (RCC). A tissue microarray study. Eur Urol Suppl 2007; 6: 32.

43. Azumi N, Battifora $\mathbf{H}$. The distribution of vimentin and keratin in epithelial and nonepithelial neoplasms. A comprehensive immunohistochemical study on formalin- and alcohol-fixed tumors. Am J Clin Pathol 1987; 88 (3): 286-926.

44. Bazille C, Allory Y, Molinie V, Vieillefond A, Cochand-Priollet B, Cussenot $\mathrm{O}$ et al. Immunohistochemical characterisation of the main histologic subtypes of epithelial renal tumours on tissue-microarrays: study of 310 cases. Ann Pathol 2004; 24 (5): 395-406.

45. Young AN, Amin MB, Moreno CS, Lim SD, Cohen C, Petros JA et al. Expression profiling of renal epithelial neoplasms: a method for tumor classification and discovery of diagnostic molecular markers. Am J Pathol 2001; 158 (5): 1639-1651.

46. Pan CC, Chen PC, Ho DM. The diagnostic utility of MOC31, BerEP4, RCC marker and CD10 in the classification of renal cell carcinoma and renal oncocytoma: an immunohistochemical analysis of 328 cases. Histopathology 2004; 45 (5): 452-459.

47. Mazal PR, Exner M, Haitel A, Krieger S, Thomson RB, Aronson PS et al. Expression of kidney-specific cadherin distinguishes chromophobe renal cell carcinoma from renal oncocytoma. Hum Pathol 2005; 36 (1): 22-28.

48. Williams A, Higgins J, Zhao H, Ljungberg B. Brooks JD. CD 9 and vimentin distinguish clear cell from chromophobe renal cell carcinoma. BMC Clin Pathol 2009; 18 (9): 1472-1483.

49. McGregor DK, Khurana KK, Cao C, Tsao CC, Ayala G, Krishnan B et al. Diagnosing primary and metastatic renal cell carcinoma: the use of the monoclonal antibody. Renal cell carcinoma marker. Am J Surg Pathol 2001; 25 (12): 1485-1492.

50. Abrahams NA, MacLennan GT, Khoury JD, Ormsby AH, Tamboli P, Doglioni C et al. Chromophobe renal cell carcinoma: a comparative study of histological, immunohistochemical and ultrastructural features using high throughput tissue microarray. Histopathology 2004; 45 (6): 593-602.

51. Lin F, Zhang PL, Yang XJ, Shi J, Blasick T, Han WK et al. Human kidney injury molecule-1 (hKIM-1): a useful immunohistochemical marker for diagnosing renal cell carcinoma and ovarian clear cell carcinoma. Am J Surg Pathol 2007; 31 (3): 371-381.

52. Ingold B, Wild PJ, Nocito A, Amin MB, Storz M, Heppner FLet al. Renal cell carcinoma marker reliably discriminates central nervous system haemangioblastoma from brain metastases of renal cell carcinoma. Histopathology 2008; 52 (6): 674-681.

53. Williams GM, Lynch DT. Renal Oncocytoma. Stat Pearls [Internet]. Last Update: April 4, 2019.

54. Buchner A, Castro M, Hennig A, Assmann G, Hofstetter A, Stief C, Zimmermann W. Identification of an independent prognostically relevant gene signature in metastatic renal cell carcinoma using laser microdissection and oligonucleotide microarrays. Eur Urol Suppl 2007; 6: 32.

Received May 2, 2020. Accepted May 31, 2020. 